\title{
Natural Landscape-Friendly Animation Display Technique Using Shape-Changing Artificial Grass System*
}

\author{
Kojiro Tanaka ${ }^{12}$, Masahiko Mikawa ${ }^{1}$ and Makoto Fujisawa ${ }^{1}$
}

\begin{abstract}
This paper introduces a display technique to show gradation animation using a shape-changing artificial grass system. Recently, liquid crystal displays and projectors have been used in a natural public facility, such as a park, but their artificial appearance can spoil the natural scenery. The proposed method is useful for developing a natural landscapefriendly display. We developed a grass pixel system to display the gradation. The grass pixel consists of yellow and green grass, and the length of the green grass moves vertically. The grass pixel uses a pin-based shape-changing system using a stepper motor to control the grass length dynamically. We evaluated the grass pixel gradation using an image processing of HSV (Hue, Saturation, Value of Brightness) values. In the measurement, the pixel system moved the grass length from minimum to maximum, and we took images of the running grass pixel from several angles. As a result, we observed that the HSV values changed linearly with the movement of the length of the green grass. We also developed a $3 \times 3$ pixels grass display, which can demonstrate several gradation animations.
\end{abstract}

\section{INTRODUCTION}

Recently, computer graphics (CG) have been widely used in a natural environment, such as a park, for signage and entertainment. For example, there was an art projection mapping project in the river [1], and the zoo park uses digital signage for the zoo's visitors [2]. When CG is used outside, liquid crystal displays (LCDs) and projectors usually show CG images. However, these devices spoil the natural landscape because they look like artificial materials. Thus, natural spot needs a display device that can blend into natural scenery.

To solve this problem, we focus on grass art that displays images by changing the grass shape. People usually enjoy grass art as entertainment. Currently, grass art has attracted significant attention as a natural public display. Many researchers have developed systems that can display CG images on the lawn. The grass systems can show static CG image [3][4]. However, there are few grass display techniques that can play an animation. Displaying the animation is essential as the public display.

We propose a natural landscape-friendly grass display technique that can show the gradation animation by changing the grass shape smoothly as shown in Fig. 1. In this paper, we develop a grass pixel consisting of yellow and green artificial grass. The pixel system can provide the grass gradation dynamically by moving the length of the green grass. The grass pixel system uses a pin-based shape-changing system using

*This work was not supported by any organization

1 Graduate School of Comprehensive Human Sciences, University of Tsukuba, 1-2, Kasuga, Tsukuba, Ibaraki, 305-8550, Japan

2 tanaka.kojiro.sp@alumni.tsukuba.ac.jp a stepper motor to control the grass length. We measured the gradation of the grass pixel system using an image processing of HSV values (Hue, Saturation, Value of Brightness). In addition, we developed a $3 \times 3$ pixels grass display using multiple grass pixels, and the display can play three types of example gradation animation as demonstrations.

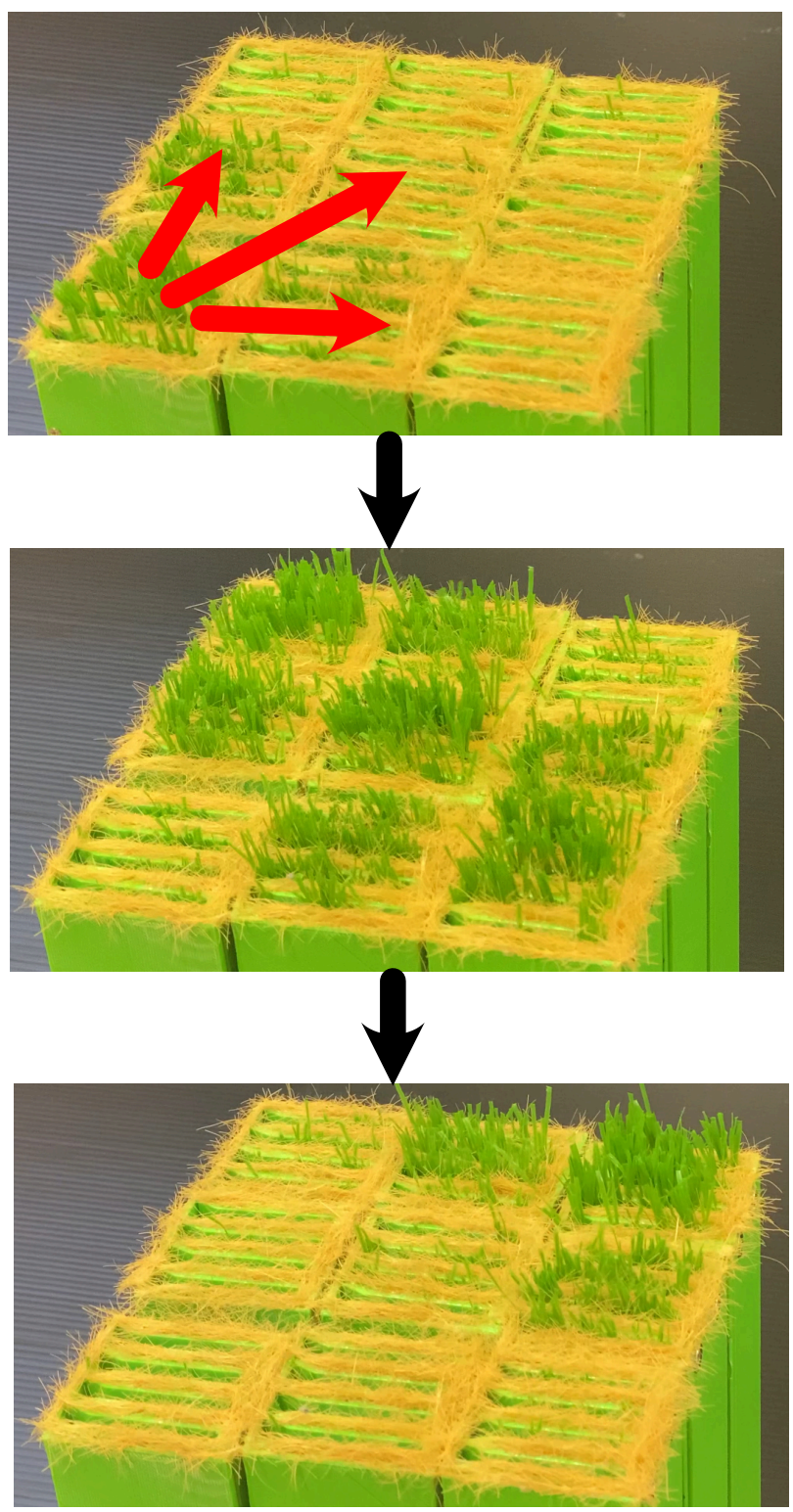

Fig. 1. Gradation animation on the proposed grass system 


\section{RELATED WORK}

\section{A. Grass Art as Display}

Grass art has been essential as entertainment and public display in nature field. The popularity of grass display research is gradually growing. Ackroyd \& Harvey presented a grass art that can display a photograph [4]. The art shows that the grass can display the gradation image. Sugiura et al. proposed a method that can print a simple binary image on the lawn [3]. This method allows a user to draw a static image on the lawn easily. Scheible et al. developed an approach that the user can make a large grass art as the organic display using a drone [5]. However, these display techniques can only show static images. Thus, there are few grass display methods that can give the animation with the gradation.

\section{B. Grass-Based Smart Material Interface}

To express the animation on the lawn, a system that can change the shape of grass is needed. Recently, Vyas et al. proposed a novel interface called the smart material interface that focuses on the shape or color of materials [6]. There are several studies on grass-based smart material interfaces. Minuto et al. presented an interface that can move each individual artificial grass sequentially [7]. Umezu et al. developed a hair top-based shape-changing interface using an LCD brightness [8]. These methods cannot change the gradation on the lawn pixel by pixel quickly.

\section{Image Displays Using Natural Materials}

Displays using natural materials can provide living environment-friendly images. Sugiura et al. proposed a fur display to show the static image by controlling the tips of the fur [9]. Nagafuchi et al. presented a drawing technique on the ground using water [10]. Robinson et al. produced a small robot printer that can draw a static image using everyday foodstuffs [11]. In the art fields, Rozin presented the display art that can show the gradation animation using wooden materials [12]. Thus, many researchers and artists are developing nature-friendly displays for various purposes for the real world. In this paper, we investigate and develop a grass animation display that can blend into the natural public area.

\section{CONCEPT}

In this paper, we focus on the grass gradation depending on the difference between the length of lively green grass and dying yellow grass as shown in Fig. 2. The green grass grows, and it gets taller day by day. However, dying yellow grass stops growing, and its length cannot be changed. The lawn of the yellow grass has only yellow surface. The more the green grass grows, the lawn gets greener.

We designed a grass pixel system that can change the grass gradation by controlling the grass length. Fig. 3 shows the overview of the grass system. The grass system's surface is planted with artificial yellow grass. In the pixel system, there is a moving pin with artificial green grass. This pin system was designed based on pin-based shape-changing display techniques. When the pin moves vertically, the green grass moves in and out between the gaps of the yellow grass, as if the grass is growing and dying. Then, the grass system can control the pixel-based gradation smoothly. Thus, the display system can play the grass animation since the pin can run quickly.

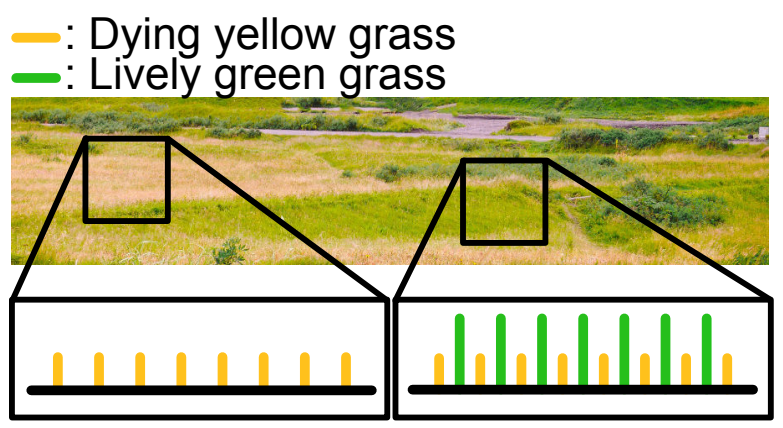

Fig. 2. Grass gradation by yellow and green grass

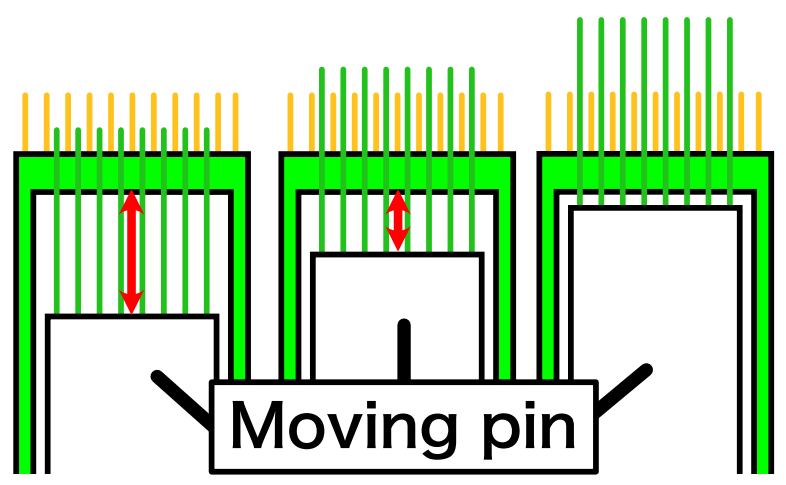

Fig. 3. Concept of the grass display technique

\section{IMPLEMENTATION}

\section{A. Hardware}

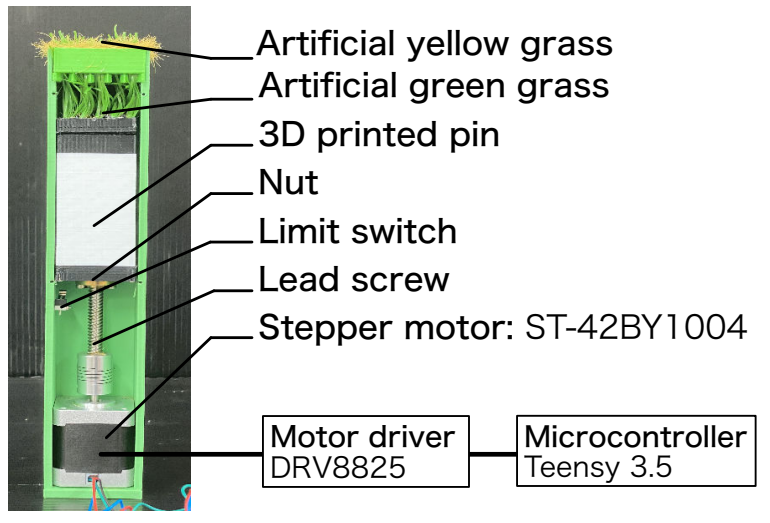

Fig. 4. Hardware configuration of grass pixel

Fig. 4 illustrates the hardware system of the grass pixel. The dimensions are $50.0(\mathrm{~L}) \times 50.0(\mathrm{~W}) \times 275.0(\mathrm{H})[\mathrm{mm}]$. The pixel system consists of a stepper motor (ST-42BY1004, 400 [step/rotation]), a lead screw with a nut (length: 100 [mm], pitch: 2 [mm], lead: 8 [mm]), a limit switch, a threedimensional (3D) printed pin (size: $48.0(\mathrm{~L}) \times 48.0(\mathrm{~W})$ 


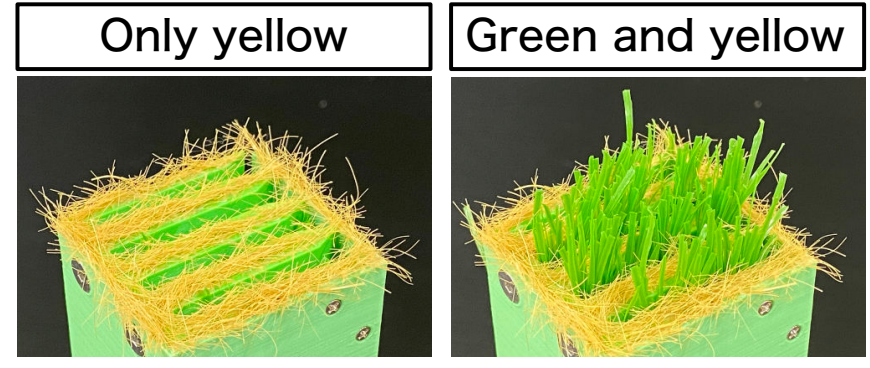

Fig. 5. Slits for moving green grass

$\times 100.0(\mathrm{H})[\mathrm{mm}])$, artificial green grass (length: $50[\mathrm{~mm}]$ ), and artificial yellow grass (length: 10 [mm]). The lead screw connects the $3 \mathrm{D}$ printed pin through the nut, and the limit switch is attached to the inner wall of the outer case of the grass pixel. The green grass is installed on the 3D printed pin, and the yellow grass is fixed on the top of the outer case. As shown in Fig. 5, the surface of the yellow grass has some slits, and the green grass can go in and out the slits. A microcontroller (Teensy 3.5) controls the stepper motor through a motor driver (DRV8825).

\section{B. Software}

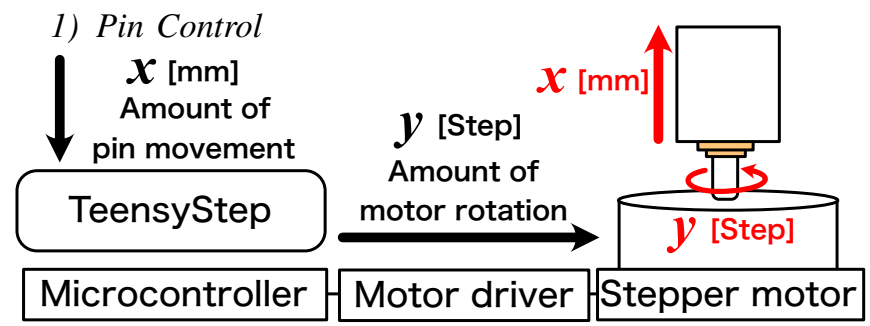

Fig. 6. Software system for moving pin

The grass pixel system controls the length of the grass by moving the pin vertically. The stepper motor allows the pin to run, and the microcontroller needs to send the steps to the motor. Fig. 6 shows the moving pin system. The system uses a stepper motor management software (TeensyStep [a]). The software can convert an amount of pin movement to the steps. The controller gives the output steps to the grass pixel through the motor driver, and the motor rotates the lead screw to move the pin according to the received data. Finally, the length of the green grass can be controlled smoothly. In the system, the stepper motor can move the pin $8 \mathrm{~mm}$ per rotation. Hence, the grass length can be moved from 0 to 24 [mm] since the motor can rotate from 0 to 3 [rotation].

\section{2) Animation System}

The grass gradation pixel can change between frames and play the animation. Fig. 7 illustrates the overview of the grass animation system. Four levels of the gradation values are the input data of the animation system as keyframes. The keyframe data is set at the same time interval. As shown in Fig. 7, the control values, the 0th, 3rd, 2nd, and 1st level of the gradation are input into the grass pixel in turn. Four types of grass lengths $(0,8,16$, and $24[\mathrm{~mm}])$ are assigned for the four levels of the gradation as shown in Fig. 8. The

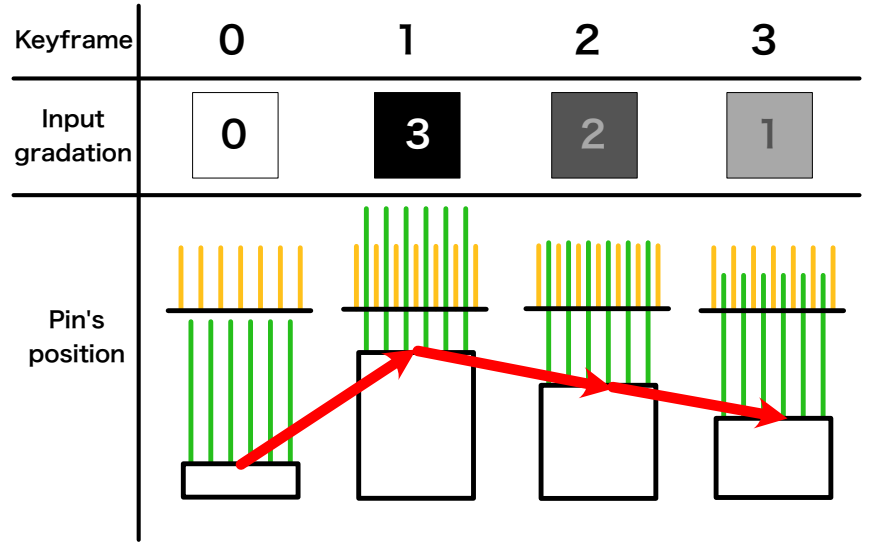

Fig. 7. Animation system of grass pixel
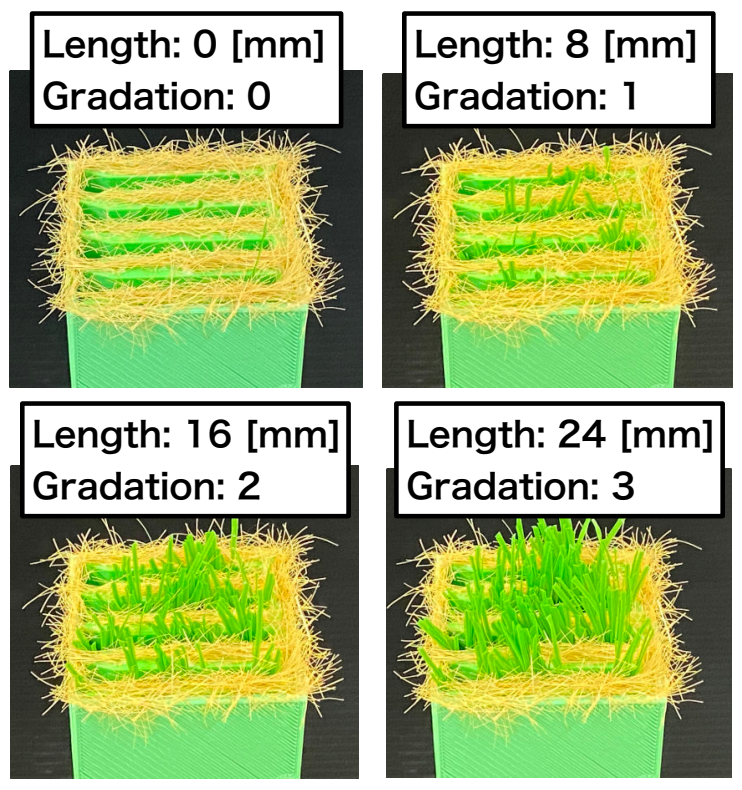

Fig. 8. Grass length corresponded with four levels of gradation

grass length is set at each frame according to the gradation values, and the pin moves to change the grass height. As a result, the grass pixel can play the gradation animation, and the grass display consisting of multiple grass pixels can show the gradation images.

\section{GRADATION EVALUATION}

The grass pixel gradation changes according to the additive color mixing by light reflection. The slits on the top of the grass pixel affect the additive color mixing. To clarify the effects, we conducted several simple evaluations of the pixel's gradation through image processing. Fig. 9 illustrates an experimental environment. The experiment used an iPhone11 Pro as a camera (12 MP, f/1.8, $26 \mathrm{~mm}$, wide-angle lens, 1080 p, $60 \mathrm{fps}$ ), and the camera's exposure, ISO value, and white balance were fixed. The light sources of the environment were fluorescent lamps. The camera's height was $0.975[\mathrm{~m}]$ from the grass pixel plane, and the distance between the camera and target pixel was $1.65[\mathrm{~m}]$. Since it is necessary 


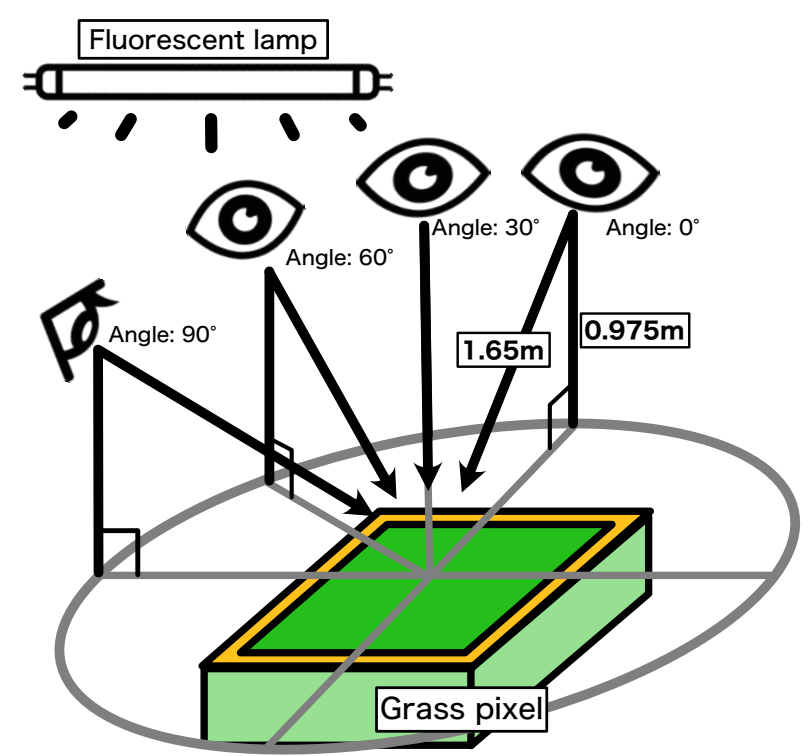

Fig. 9. Grass gradation evaluation environment

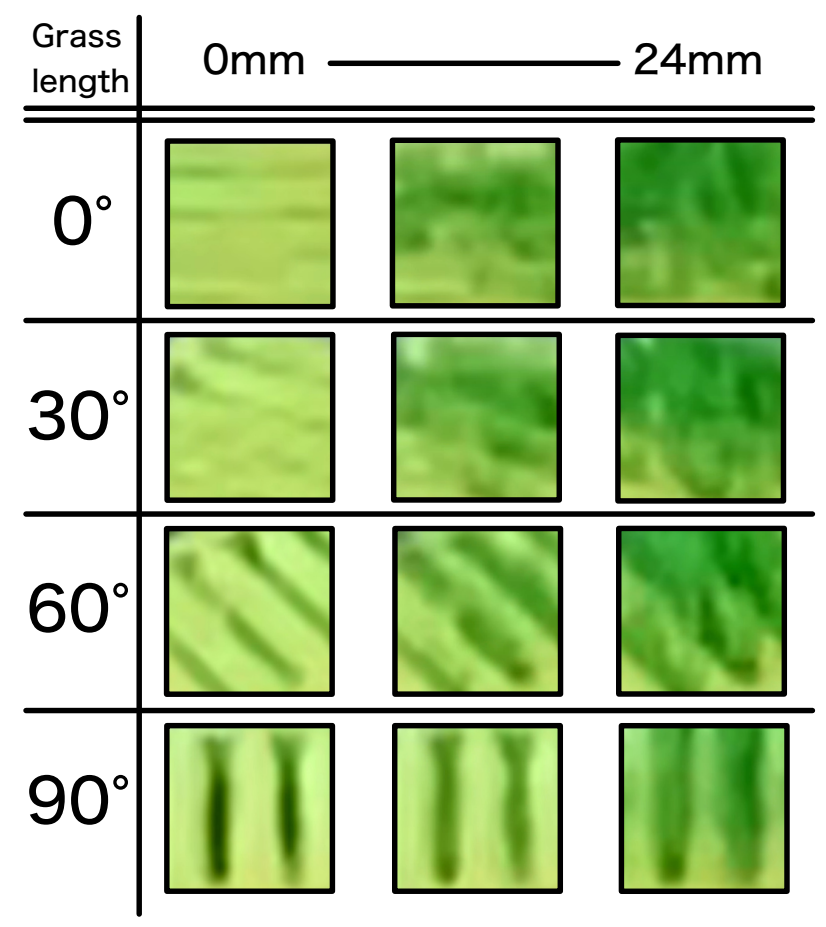

Fig. 10. Grass pixel surface from four angles

to take a lot of images from various angles using the camera to observe the effects of the slits, the camera was rotated around the grass pixel every $30^{\circ}$ from $0^{\circ}$ to $90^{\circ}$. The angle between the camera and slits was perpendicular at $0^{\circ}$, and the angle was parallel at $90^{\circ}$. When the camera captured the images of the grass pixel, the grass length was moved from 0 to 24 [mm]. Only the top surface of the grass was cropped using the digital zoom function as shown in Fig 10.

To measure the grass gradation at each angle, the average values of $\mathrm{H}, \mathrm{S}$, and $\mathrm{V}$ of each image in the captured video were calculated using OpenCV [b]. Fig. 11 shows the
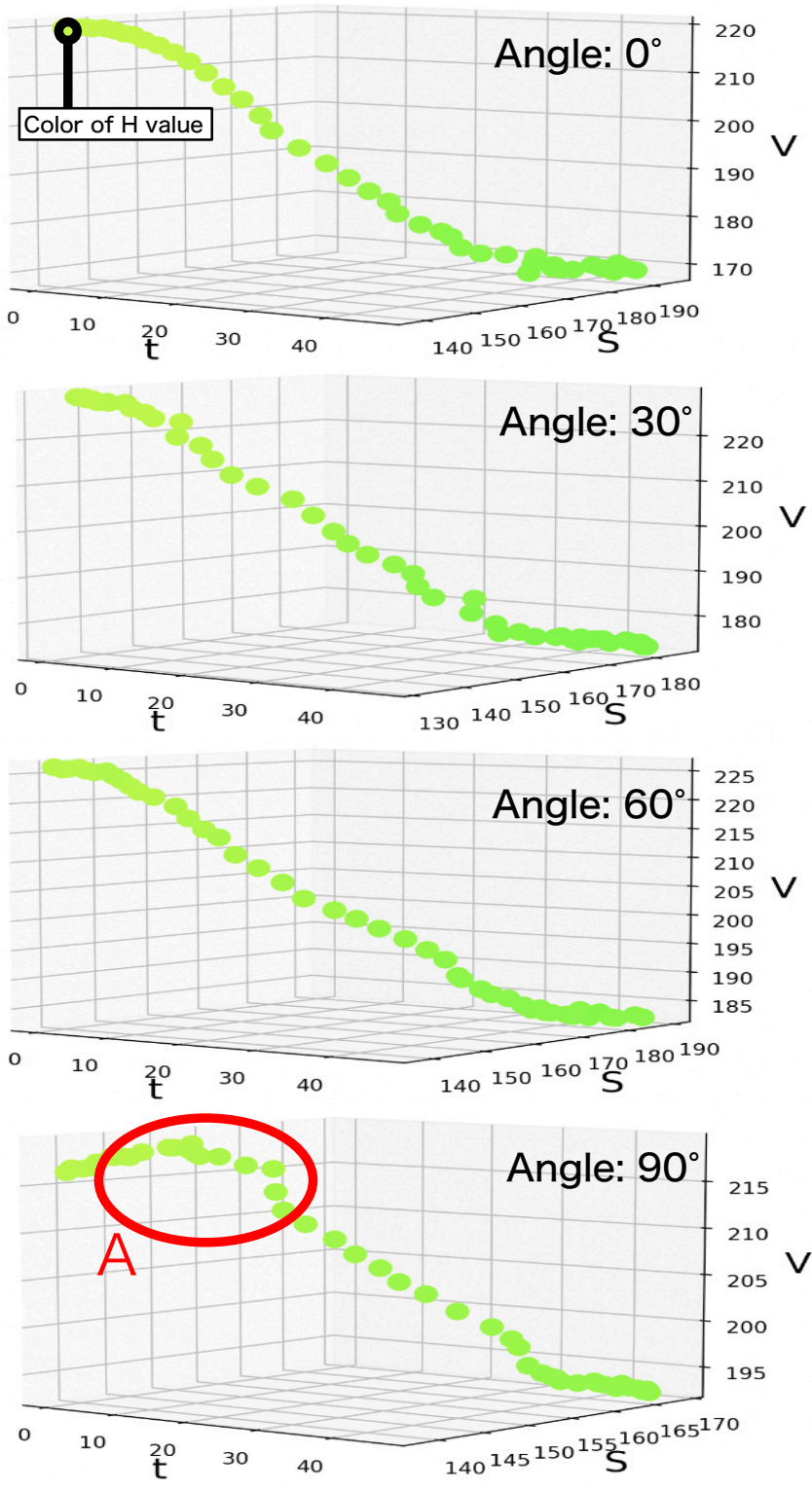

Fig. 11. Image processing results of HSV values

variations of the HSV values with the time-series frame. From the top, the time variations of the HSV values at $0^{\circ}, 30^{\circ}, 60^{\circ}$, and $90^{\circ}$ are shown, respectively. $t$ represents the frame order in the recorded video. The $\mathrm{H}$ values are represented by the color of the dots, and range of the $\mathrm{S}$ and $\mathrm{V}$ values is from 0 to 255. As shown in Fig. 11, the variations of the HSV values with time were linear at all angles. In the results of $0^{\circ}, 30^{\circ}$, and $60^{\circ}$ angles, the ranges of $\mathrm{S}$ and $\mathrm{V}$ values were about 50 . However, the ranges of $\mathrm{S}$ and $\mathrm{V}$ values at $90^{\circ}$ were about 30 . Moreover, Fig. 11A illustrates that the part of the graph was different from the other results. We suppose that this was because the visibility of the slits on the upper surface of the grass at $90^{\circ}$ was quite different compared to other angles. As shown in the bottom row of Fig. 10, at $90^{\circ}$, the slits appeared to be clearer than in the other images, and color mixing seemed to be prevented. 


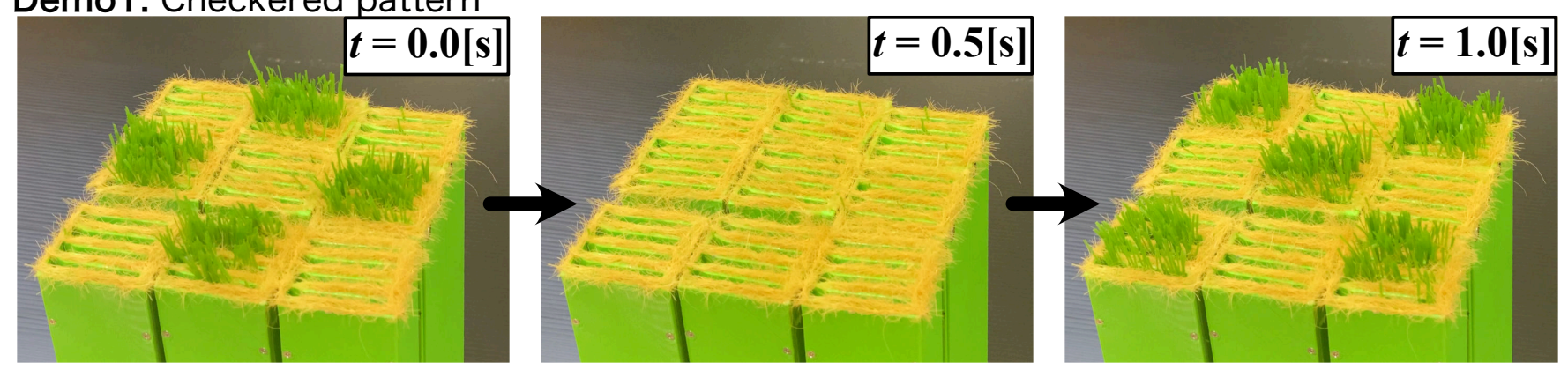

\section{Demo2: Spiral}

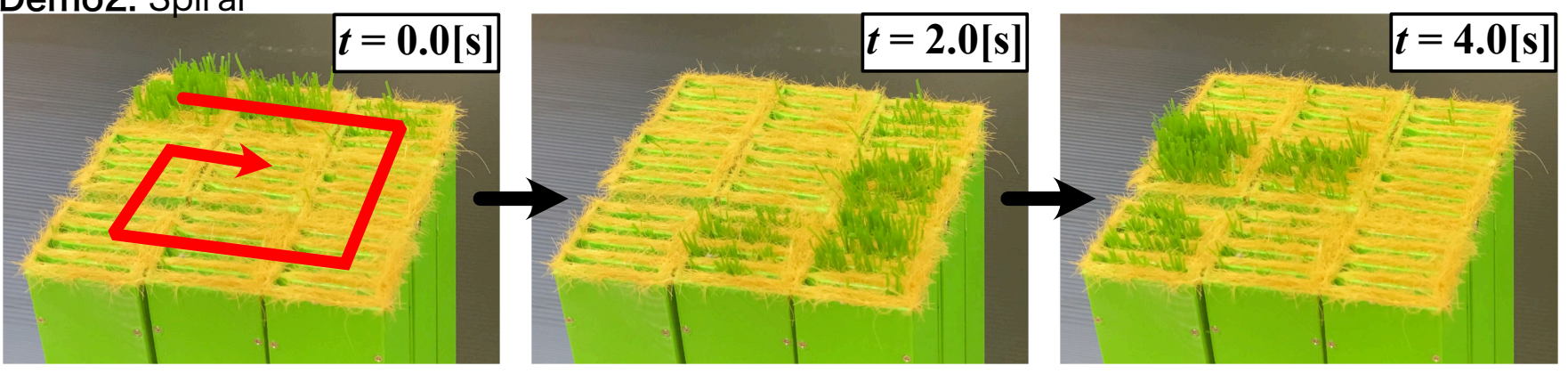

\section{Demo3: Wave}

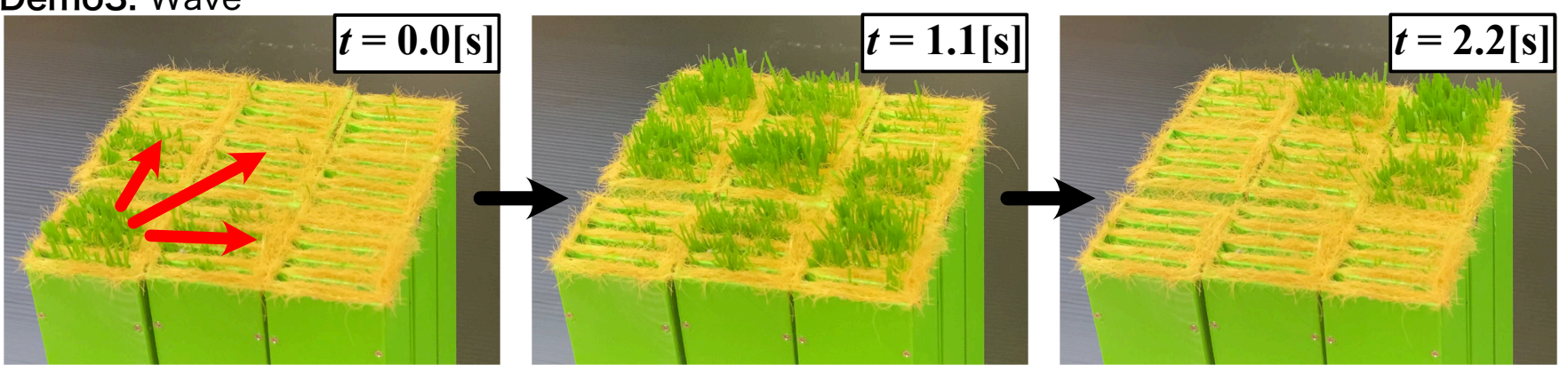

Fig. 12. Example gradation animation using $3 \times 3$ pixels grass display

\section{EXAMPLE ANIMATION}

We made the following three demonstrations to show the gradation animation using our grass system: checkered pattern (Demo1), spiral (Demo2), and wave (Demo3). In the test animations, the $3 \times 3$ pixels grass display was developed using nine grass pixels, and the input movie was $3 \times 3$ pixels with a four-gray scale. The grass display animation's keyframe interval is 0.5 [s].

The checkered patterns were displayed on the grass display in Demo1 as shown in the upper part of Fig. 12. Demo1 was created to check the grass display's ability to switch between binary images. When the grass system played the animation, the checkered images can change smoothly.

The spiral animation was used in Demo2 as shown in the middle part of Fig. 12. Demo2 was created to confirm that the grass display can draw a path. The spiral animation started from the top-left pixel of the grass display and moved clockwise toward the display center. Then, the grass display can show the path animation smoothly and clearly.

The wave animation was used in Demo3 as shown in the bottom part of Fig. 12. Demo3 was created to check that the grass display can give the entire gradation animation. In Demo3 animation, the wave started from the bottom left pixel and spread to the entire grass display.

Through these demonstrations, we confirmed that the grass display can play the gradation animation.

\section{FUTURE WORK}

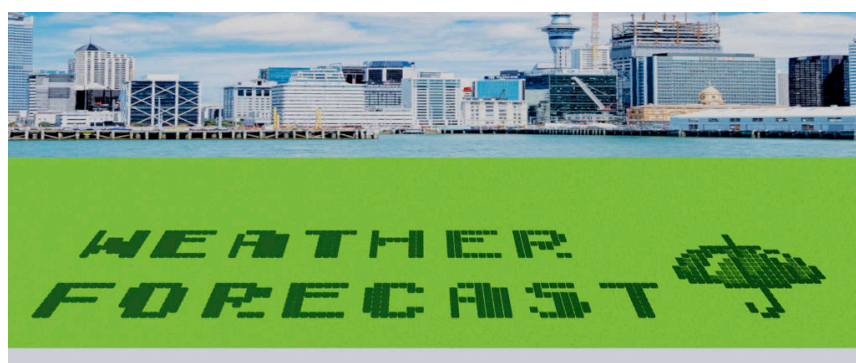

Fig. 13. Future application with our proposal (Umbrella material: [c])

The proposed method can be used to develop a natural 
landscape-friendly display in the public space, such as a park. Fig. 13 illustrates a weather forecast application using the grass display. In the application, the lawn displays the text and umbrella, thus, the natural public area is clean. We believe that the grass display can be applied to several natural environment-friendly applications, such as guide maps advertisements in the park.

\section{LIMITATIONS}

\section{A. Speed and Resolution of Grass Display}

In Section VI, the animation keyframe interval is 0.5 [s]. When the interval is smaller than 0.5 [s], the stepper motor of the grass pixel cannot synchronize with the other motors well. In addition, the current grass display can show several $3 \times 3$ pixels animation. However, the system requires many controllable grass pixels to show the text and character animation on the grass. The grass display needs a stable motor system that can operate many motors simultaneously to play the graphical animation smoothly.

\section{B. Outdoor Experiment}

Currently, the grass gradation was evaluated only indoors and not outdoors. When the grass display is used in a playground outside, it is necessary to consider how to install the grass system outdoors.

\section{User Evaluation}

In this paper, the grass pixel gradation was evaluated quantitatively and objectively through image processing using OpenCV. The conditions of the evaluation experiments were simple; hence, we will complicate the conditions and proceed with the quantitative evaluation. Furthermore, quantitative user evaluations should be conducted to improve the grass display.

\section{CONCLUSIONS}

We proposed a natural landscape-friendly animation display technique using a shape-changing artificial grass system. In this paper, we created a grass pixel system consisting of artificial yellow and green grass. The grass system displayed the gradation by moving the length of the green grass dynamically using a pin-based shape-changing system. We measured the gradation display performance of the grass pixel through an image processing of HSV values. As a result, the HSV values could change linearly when the grass length increased. We created a $3 \times 3$ pixels grass display to confirm the display's animation performance. The grass display played three animations. In the future study, we will develop a stable system that can control many grass pixels to display a graphical smooth animation on the grass. In addition, we will evaluate the grass gradation outdoors and conduct a subjective assessment of the grass display by experimental participants.

\section{REFERENCES}

[1] Hokkaido Magazine KAI. Sosei River Park Symphony. http://kai-hokkaido.com/en/feature_vol34_ichiba8/. Accessed: 2021-03-03.

[2] AIRPORT SUPPLIERS. Network Appliances for Standalone \& End-to end Digital Signage Network Solutions. https://www. airport-suppliers.com/supplier-press-release/ network - appliances - for - standalone - end - to - end - digital signage-network-solutions-5/. Accessed: 2021-04-07.

[3] Yuta Sugiura et al. "Grassffiti: Drawing Method to Produce Large-Scale Pictures on Conventional Grass Fields". In: Proceedings of the Eleventh International Conference on Tangible, Embedded, and Embodied Interaction. TEI '17. Yokohama, Japan: Association for Computing Machinery, 2017, pp. 413-417. ISBN: 9781450346764.

[4] Ackroyd \& Harvey. Big Chill. https://www.ackroydandharvey.com/big-chill/. Accessed: 2021-03-18.

[5] Jürgen Scheible and Markus Funk. "DroneLandArt: Landscape as Organic Pervasive Display". In: Proceedings of the 5th ACM International Symposium on Pervasive Displays. PerDis '16. Oulu, Finland: Association for Computing Machinery, 2016, pp. 255-256. ISBN: 9781450343664.

[6] Dhaval Vyas et al. "Smart Material Interfaces: A New Form of Physical Interaction”. In: CHI '12 Extended Abstracts on Human Factors in Computing Systems. CHI EA '12. Austin, Texas, USA: Association for Computing Machinery, 2012, pp. 1721-1726. ISBN: 9781450310161.

[7] Andrea Minuto and Anton Nijholt. "Growing Grass: A Smart Material Interactive Display, Design and Construction History". In: Proceedings of the 1st Workshop on Smart Material Interfaces: A Material Step to the Future. SMI '12. Santa Monica, California: Association for Computing Machinery, 2012. ISBN: 9781450315180.

[8] Shuhei Umezu et al. "Hairlytop Interface: A Basic Tool for Active Interfacing". In: Proceedings of the Adjunct Publication of the 27th Annual ACM Symposium on User Interface Software and Technology. UIST'14 Adjunct. Honolulu, Hawaii, USA: Association for Computing Machinery, 2014, pp. 95-96. ISBN: 9781450330688.

[9] Yuta Sugiura et al. "Graffiti Fur: Turning Your Carpet into a Computer Display". In: Proceedings of the 27th Annual ACM Symposium on User Interface Software and Technology. UIST '14. Honolulu, Hawaii, USA: Association for Computing Machinery, 2014, pp. 149-156. ISBN: 9781450330695.

[10] Reona Nagafuchi et al. "Polka: A Water-Jet Printer for Painting on the Grounds". In: Proceedings of the International Conference on Advanced Visual Interfaces. AVI '20. Salerno, Italy: Association for Computing Machinery, 2020. ISBN: 9781450375351.

[11] Simon Robinson et al. "Sustainabot - Exploring the Use of Everyday Foodstuffs as Output and Input for and with Emergent Users". In: Proceedings of the 2019 CHI Conference on Human Factors in Computing Systems. CHI '19. Glasgow, Scotland Uk: Association for Computing Machinery, 2019, pp. 1-12. ISBN: 9781450359702.

[12] Daniel Rozin. Wooden Mirror. http://www.smoothware.com/danny/woodenmirror.html. Accessed: 2021-04-09.

\section{SUPPORTING ONLINE MATERIALS}

[a] Lutz Niggl. TeensyStep. https://luni64.github.io/TeensyStep/. Accessed: 2021-03-05.

[b] OpenCV team. OpenCV. https://opencv.org. Accessed: 2021-04-08.

[c] blue-sky. SKY's animation. https://pata2.jp/127/sky2.html. Accessed: 2021-04-12. 